\title{
The reliability horizon for semi-classical quantum gravity: Metric fluctuations are often more important than back-reaction
}

\author{
Matt Vissert \\ Physics Department, Washington University, St. Louis, Missouri 63130-4899
}

(gr-qc/9702041; 20 February 1997; Revised 18 June 1997)

\begin{abstract}
In this note I introduce the notion of the "reliability horizon" for semi-classical quantum gravity. This reliability horizon is an attempt to quantify the extent to which we should trust semi-classical quantum gravity, and to get a better handle on just where the "Planck regime" resides. I point out that the key obstruction to pushing semi-classical quantum gravity into the Planck regime is often the existence of large metric fluctuations, rather than a large back-reaction. There are many situations where the metric fluctuations become large long before the back-reaction is significant. Issues of this type are fundamental to any attempt at proving Hawking's chronology protection conjecture from first principles, since I shall prove that the onset of chronology violation is always hidden behind the reliability horizon.

Revisions: Central definitions and results essentially unchanged. Discussion of the relationship between this letter and the Kay-Radzikowski-Wald singularity theorems greatly extended and clarified. Discussion of reliability horizon near curvature singularities modified. Several references added. Minor typos fixed. Technical $\mathrm{T}_{\mathrm{E}} \mathrm{X}$ modifications.
\end{abstract}

\section{INTRODUCTION}

Semi-classical quantum gravity [1] is the approximation wherein we keep the gravitational field classical, while quantizing everything else. This hybrid theory is clearly a half-way-house on the road to quantum gravity, but it has two very decided advantages over the various speculative theories of quantum gravity currently in vogue: (1) Semi-classical quantum gravity is firmly based in experimental reality, and (2) Semi-classical quantum gravity is relatively tractable. In particular, essentially all of black hole thermodynamics, the stress-energy renormalization programme [2,3], and Lorentzian wormhole physics [4 8], is carried out within this framework.

A related issue that is currently only treatable within the framework of semi-classical quantum gravity, and that has excited much recent attention, is that of Hawking's chronology protection conjecture [9, 10]. Various attempts at proving this conjecture have been made [11 14, culminating in the singularity theorems of Kay, Radzikowski, and Wald [15]. These theorems are set up, proved, and in fact only make sense within the semi-classical quantum gravity approximation, so it behooves us to develop a rather good feel for where we should stop believing this approximation. Qualitatively, the answer to this question has been known since the pioneering work of Wheeler in the late 1950's [16,17]: We should certainly stop believing semi-classical quantum gravity once we enter the Planck regime. The subtleties arise in recognizing the onset of Planck scale physics.

(Of course there is the logical possibility, however unlikely, that all current theories could break down with only just a little more energy in our accelerators. I will not deal with any new physics that may be hiding just around the corner, but will instead focus on the region where we know that current theories must break down of their own accord even if no other new physics intervenes at lower energies.)

To help get a better handle on these issues I will introduce a few new concepts: the "reliable region"; the "reliability boundary"; and the "reliability horizon". These concepts will be defined in a way that is similar to standard concepts of classical general relativity: the chronology-violating region; chronology boundary; and chronology horizon respectively. The precise location of reliability horizon I define is (deliberately) somewhat fuzzy, in many respects it is similar to the stretched horizon of the black hole Membrane Paradigm [18]. We can always argue over the last factor of ten or so concerning where exactly to place the reliability horizon. This fuzziness is not a problem and the notion of reliability horizon is still useful since I shall show that the reliability horizon is always outside the chronology horizon.

Physically, the reliability horizon is characterized by the onset of either large metric fluctuations (Planck scale curvature fluctuations) or a large back-reaction (Planck scale expectation value for the curvature). I shall argue that in many situations the onset of large metric fluctuations precedes the onset of large back-reaction. In view of this we should stop believing the semi-classical quantum gravity approximation at the reliability horizon, before reaching the chronology horizon.

\section{THE CHRONOLOGY HORIZON}

Let $\gamma$ be any geodesic (spacelike, null, or timelike) that connects some point $x$ to itself. Let $\sigma_{\gamma}(x, y)$ denote the relativistic interval from $x$ to $y$ along the geodesic $\gamma$.

$$
\sigma_{\gamma}(x, y)=\left\{\begin{array}{cc}
+s^{2} & \text { if the geodesic is spacelike, } \\
0 & \text { if the geodesic is lightlike, } \\
-\tau^{2} & \text { if the geodesic is timelike. }
\end{array}\right.
$$

Define level sets $\Omega\left(\ell^{2}\right)$ by 


$$
\Omega\left(\ell^{2}\right) \equiv\left\{x: \exists \gamma \neq 0 \mid \sigma_{\gamma}(x, x) \leq \ell^{2}\right\} .
$$

If the metric were Riemannian, rather than Lorentzian, these level sets would be very simple. In particular we would have $\Omega(0)=\emptyset$. Because the metric is indefinite the set $\Omega(0)$ has the possibility of being nontrivial. Note that it is essential that the geodesic in question not be the trivial geodesic from $x$ to itself, otherwise $\Omega\left(\ell^{2}\right)$ would trivially be the whole spacetime for $\ell^{2} \geq 0$.

The set $\Omega(0)$ is called the "chronology violating region". By definition any $x \in \Omega(0)$ is connected to itself by a nontrivial timelike or null geodesic - as such we clearly have the possibility of time travel from the point $x$ to itself.

The set $\mathcal{B} \equiv \partial[\Omega(0)]$ is the "chronology boundary"this is the boundary that we will have to cross in order to actively participate in time travel effects.

The set $\mathcal{H}^{+} \equiv \partial\left[J^{+}(\Omega(0))\right]$ is the "chronology horizon"-it is the boundary of the future of the chronology violating region. This is the boundary that we will have to cross in order to passively participate in time travel effects. (Passive participation is the ability to see time travel effects somewhere in one's past, without being able to influence the past. This is quite sufficient to thoroughly disrupt known physics and is just as reprehensible as active participation.)

Note that these definitions only make sense if we are dealing with a fixed Lorentzian geometry - other interesting quantum field theoretic processes may be going on, but the geometry is fixed and unquantized. This is exactly the statement that we are dealing with semiclassical quantum gravity. These definitions are fundamental to analyses of the chronology protection conjecture: The basic idea is that something must go wrong on or near the chronology horizon.

\section{CHRONOLOGY PROTECTION THEOREMS}

Working entirely within the context of semi-classical quantum gravity, Kay, Radzikowski, and Wald 15 showed that in any spacetime containing a compactly generated chronology horizon there must exist certain points on the chronology horizon such that the two-point function is not locally of Hadamard form. (Similar results hold for a broad class of non-compactly generated chronology horizons.)

This is to be contrasted with the observations of Sushkov 19,20], Krasnikov 21,22], and the present author 23]. For instance, Sushkov [19] showed that in Misner space, a particularly simple exemplar for a spacetime with chronology horizon [not compactly generated], a specific choice of quantum field theory coupled with a specific choice of quantum state keeps the stress-energy regular all the way to the chronology horizon. In addition, Krasnikov [21] exhibited several $(1+1)$-dimensional geometries with bounded stress-energy near the chronology horizon (Cauchy horizon). Finally, the present au- thor has shown that with enough wormholes it is possible to keep the stress energy arbitrarily mild arbitrarily near the chronology horizon [23].

The Sushkov and Krasnikov results can be brought into conformity with the Kay-Radzikowski-Wald (KRW) results by noting that the KRW results show only that the stress-energy is "singular" at some points on the chronology horizon - and that the word singular in this context can either mean infinite or may mean simply mean undefined [15,24].

Additional investigations bearing on this matter are those of Boulware [25], Grant [26], and Tanaka and Hiscock 27].

The KRW results are formulated as stress-energy singularity theorems in semi-classical quantum gravity without back-reaction. It is only after we add back-reaction (by insisting that the spacetime satisfy the semi-classical Einstein equations) that we begin to see the outlines of a chronology protection theorem: Any spacetime that satisfies the semi-classical Einstein equations must at the very least have all two-point functions locally of Hadamard form (otherwise we cannot even define the stress-energy tensor) and therefore cannot contain a compactly generated chronology horizon.

Stated differently, if we try to insert a chronology horizon into an otherwise respectable spacetime satisfying the semi-classical Einstein equations then either (1) some points on the chronology horizon are curvature singularities, or (2) the spacetime no longer has a uniquely defined geometry (because it no longer has a uniquely defined stress-energy tensor) and so is no longer even a manifold in the normal sense.

The key point to focus on here is that the KayRadzikowski-Wald singularities occur at the actual onset of chronology violation - at the chronology boundary. By a reductio ad absurdum argument [28], this may be interpreted as indicating the breakdown of semi-classical quantum gravity at the chronology horizon. Since by this argument the chronology horizon cannot be treated by semi-classical methods it follows that time machines themselves cannot be globally treated by semi-classical methods: Time machines (if they exist at all) intrinsically require the use of full quantum gravity to describe the chronology horizon. Time machines (if they exist at all) are thus intrinsically "beyond the pale" of known physics. This is an important and crucial result: it indicates that the apparently plausible manipulations typically invoked to turn a traversable wormhole into a time machine are much less plausible under closer scrutiny.

The KRW argument, while definitively indicating the breakdown of semi-classical quantum gravity at (at least some points on) the chronology horizon, unfortunately gives very limited information as to what if anything goes wrong near the chronology horizon. I shall now argue that it is possible to introduce the notion of a reliability horizon to qualitatively describe the breakdown of semiclassical quantum gravity and shall argue that we should not trust the semi-classical approximation to quantum 
gravity beyond this reliability horizon. In particular, and in agreement with this interpretation of the KRW results, we should not trust the semi-classical approximation to quantum gravity at the chronology horizon.

\section{THE RELIABILITY HORIZON}

I will define (and justify the definition of) the reliability horizon in three stages, refining the definition as we develop additional insight.

Definition 1a: Using the notation developed above, let $\mathcal{U} \equiv \Omega\left(+\ell_{\text {Planck }}^{2}\right)$ be the "unreliable region". It consists of those points $x$ that are connected to themselves by spacelike geodesics as short as, or shorter than, one Planck length.

(It is here that the fuzziness of the reliability horizon is made manifest: If you wish to argue for a safety margin by requiring the spacelike geodesics to be longer than, say, ten Planck lengths, very few people would want to argue with you. Note further that we should not blindly declare all Planck-length geodesics to be meaningless. The existence of photons, which in the geometrical optics limit are treated as particles moving along null geodesics, shows that at least some geodesics of length zero make perfectly good sense in semi-classical gravity. It is only topologically nontrivial closed geodesics that are captured by the above definition - see the physical justification for this definition below.)

The entire thrust of this definition is that it is an attempt to give an invariant and unambiguous meaning to the notion "within a Planck length of the chronology horizon", an invariant interpretation of this phrase being necessary before it is possible to decide where the Planck regime resides.

Definition 1b: Let $\mathcal{B}_{\text {Planck }} \equiv \partial[\mathcal{U}] \equiv \partial\left[\Omega\left(+\ell_{\text {Planck }}^{2}\right)\right]$ be the "reliability boundary" - this is the boundary that we will have to cross in order to actively probe the unreliable region.

Definition 1c: The set $\mathcal{H}_{\text {Planck }}^{+} \equiv \partial\left[J^{+}(\mathcal{U})\right] \equiv$ $\partial\left[J^{+}\left(\Omega\left(+\ell_{\text {Planck }}^{2}\right)\right)\right]$ is the "reliability horizon"-it is the boundary of the future of the unreliable region. This is the boundary that we will have to cross in order to passively probe the unreliable region [29].

Justification: At this stage these are merely definitions, they do not carry any weight until we physically justify the terminology. The physics behind these definitions is that I shall show, extrapolating from low energies, that at the very least the unreliable region as defined above will be subject to large fluctuations in the metric even if the expectation value of the curvature is relatively mild 30 .

(It is in addition possible that current theories break down by the introduction of new physics long before we get to the Planck scale. I am not addressing that possibility but am only interested in the unavoidable breakdown of semi-classical quantum gravity assuming that no new physics intervenes.)

To see some more technical details of the physics behind these definitions it may be helpful to consider the set

$$
\Delta \Omega=\Omega\left(+\ell_{\text {Planck }}^{2}\right)-\Omega(0) .
$$

This set consists of all points that are connected to themselves by nontrivial ultrashort Planck length spacelike geodesics, but which do not themselves suffer from the additional complication of being in the chronology violating region. Any quantum field that we try to set up in this region $\Delta \Omega$ is automatically subject to Planck scale physics.

By itself, this is not enough to justify the terminology "unreliable", but it is enough to encourage a deeper look at the issues. Continuing our preliminary analysis: If we were to think of trying to do a mode sum for the quantum field (quasi-Fourier decomposition) then the fact that in at least one direction the field has a periodicity less than $\ell_{\text {Planck }}$ implies that the mode sum will contain momenta of order $E_{\text {Planck }}$ and higher. Two cases of extremely high symmetry can be used to clarify this point: Consider a $(3+1)$-dimensional hyper-cylinder of circumference $\ell<\ell_{\text {Planck }}$. Any quantum field defined on such a hyper-cylinder can be decomposed into an infinite tower of $(2+1)$-dimensional quantum fields. These will consist of a low-energy mode coming from the translationally invariant mode plus an infinite stack of particles more massive than the Planck mass. The same sort of thing happens with a spherically symmetric traversable wormhole of throat radius less than one Planck length; the mode decomposition now runs over spherical harmonics (plus an undetermined radial mode). Again we obtain an infinite stack of trans-Planckian particles, this time $(1+1)$-dimensional particles labelled by the angular momentum quantum numbers. This is certainly enough to justify calling $\Omega\left(+\ell_{\text {Planck }}^{2}\right)$ the "trans-Planckian region".

Now as long as all the quantum field theories in question are renormalizable we should not worry about this trans-Planckian physics: after all the key aspect of renormalizable theories is that we do not need to know the details of the high energy features of the theoryrenormalizable theories are precisely those for which we can still make low energy predictions without worrying about ultra-high energy phenomena.

The key point, however, is that Einstein gravity is itself known to be non-renormalizable [16, 17]. If we had a renormalizable theory of quantum gravity then the entire discussion of the reliability horizon would be moot. The reliability horizon has to do with the breakdown of our trust in the semi-classical theory based on Einstein gravity - it is a consequence of the non-renormalizability of Einstein gravity and not a fundamental limitation on the as yet undiscovered theory of full quantum gravity.

Indeed, if we take Einstein gravity and linearize it about the background we are interested in, we can then 
ask how the linearized gravitons behave as quantum fields on this background geometry. The resulting quantum field theory is well known to be non-renormalizable with a dimensionful coupling constant given by the Planck mass [16, 17]. Once we enter the unreliable region these linearized gravitons are subject to Planck scale physics which in this non-renormalizable theory is definitely a disaster. Inside the unreliable region the linearized gravitons will be strongly interacting (and also unitarity violating) and will thereby lead to Planck scale fluctuations in the curvature, even if the expectation value of the curvature is pleasingly mild.

It is ultimately these large metric fluctuations and associated Planck scale curvature fluctuations that tell us that we should no longer trust semi-classical quantum gravity behind the reliability boundary. This is finally enough to justify calling $\Omega\left(+\ell_{\text {Planck }}^{2}\right)$ the "unreliable region".

Definition 2: An improvement of the previous definition, if the manifold in question is multiply connected one, is to keep track of the winding number of the geodesic. (In spacetimes containing traversable wormholes this will just be the number of times the geodesic threads through one of the wormholes.) Decompose the homotopy classes of self-intersecting geodesics emanating from the point $x$ into equivalence classes $\Gamma_{N}$ characterized by winding number $N$, and define

$$
\Omega_{N}\left(\ell^{2}\right) \equiv\left\{x: \exists \gamma \in \Gamma_{N} \mid \sigma_{\gamma}(x, x) \leq N^{2} \ell^{2}\right\} .
$$

The point is that if a geodesic wraps through $N$ wormholes and is of length less than $N \ell$, then at least one wormhole-to-wormhole segment of the curve must be of length less than $\ell$. Now simply replace $\Omega\left(\ell^{2}\right)$ by

$$
\Omega_{\infty}\left(\ell^{2}\right) \equiv \Omega\left(\ell^{2}\right) \cup\left(\cup_{N=1}^{\infty} \Omega_{N}\left(\ell^{2}\right)\right)
$$

in all definitions regarding the reliability region.

Definition 3: The definition given above still does not capture all of the situations in which we should cease trusting semi-classical quantum gravity. We should also not trust regions where the background manifold exhibits Planck scale curvature. We shall consider the sets

$$
\Omega_{R}\left(\ell^{2}\right)=\left\{x: R_{\mu \nu \sigma \rho} R^{\mu \nu \sigma \rho}>\ell^{-4}\right\} .
$$

Thus we shall characterize curvature singularities only by their scalar invariants.

(Unfortunately, as is well known form the theory of spacetime singularities, simply looking at the curvature invariants is not really sufficient to characterize even curvature singularities [31]. The problem lies in the fact that the Lorentzian metric is indefinite so that the curvature invariant can be zero even if the curvature tensor is not zero. This is a standard problem in the characterization of curvature singularities whose resolution requires technically messy complications beyond the scope of this letter.)
With the above definition $\Omega_{R}(0)$ is the set of curvatureinvariant singularities of the spacetime, and $\Omega_{R}\left(\ell_{P}^{2}\right)$ is a set of points for which at least one component of the Riemann tensor takes on Planck scale values.

We now augment the unreliable region by setting

$$
\mathcal{U} \equiv \Omega_{\infty}\left(+\ell_{\text {Planck }}^{2}\right) \cup \Omega_{R}\left(+\ell_{\text {Planck }}^{2}\right) .
$$

Similarly, the reliability boundary becomes

$$
\begin{aligned}
\mathcal{B}_{\text {Planck }} & \equiv \partial[\mathcal{U}], \\
& \equiv \partial\left[\Omega_{\infty}\left(+\ell_{\text {Planck }}^{2}\right) \cup \Omega_{R}\left(+\ell_{\text {Planck }}^{2}\right)\right],
\end{aligned}
$$

and the reliability horizon becomes

$$
\begin{aligned}
\mathcal{H}_{\text {Planck }}^{+} & \equiv \partial\left[J^{+}(\mathcal{U})\right], \\
& \equiv \partial\left[J^{+}\left(\Omega_{\infty}\left(+\ell_{\text {Planck }}^{2}\right) \cup \Omega_{R}\left(+\ell_{\text {Planck }}^{2}\right)\right)\right] .
\end{aligned}
$$

\section{ARE THE CHRONOLOGY PROTECTION THEOREMS PHYSICALLY RELIABLE?}

The application to the chronology protection theorems is immediate: If you interpret the KRW singularity theorems as chronology protection theorems (via the reductio ad absurdum argument [28]) then you deduce the breakdown of semi-classical quantum gravity at the chronology horizon.

Since the chronology horizon is always, by definition, inside the reliability horizon I have just defined, I would in addition argue that we should never trust the physical applicability of semi-classical quantum gravity at or near the chronology horizon. Despite the fact that the expectation value of the stress energy can be made arbitrarily small at and near the chronology horizon [19 23], the analysis of this note argues that metric fluctuations will become Planck scale once one crosses the reliability horizon.

To prove a physically trustworthy version of chronology protection, we would need either: (1) a theorem within the context of semi-classical quantum gravity that makes reference only to physics outside the reliability horizon, or (2) a theorem within the context of full-fledged quantum gravity.

Unfortunately, an acceptable theory of full-fledged quantum gravity does not yet exist, and (as mentioned above) for the approximate hybrid theory called semiclassical quantum gravity there are known to be many classes of geometries for which back reaction is negligible all the way up to the reliability horizon [19, 21, 23], in fact we can keep the back reaction negligible up to the chronology horizon. (In these situations it is the onset of large fluctuations in the metric which characterizes the reliability horizon.) The fact that any such geometry exists shows that option (1) above is impossible, and leaves us contemplating all the complications of full quantum gravity itself. 
This is compatible with the viewpoint [28] wherein one views the KRW singularity theorems as providing a reductio ad absurdum disproof of the reliability of semiclassical quantum gravity at the chronology horizon. The new features here are that the reliability horizon gives us qualitative control over where this breakdown occurs and that I have exhibited plausible physical mechanisms behind this breakdown.

This does not imply that we have succeeded in completely circumventing chronology protection-I do not mean to imply that this analysis shows that we can actually succeed in building a time machine.

What the this letter does is to drive home the point that semi-classical quantum gravity is fundamentally incapable of answering the question of whether or not the universe is chronology protected. To really answer this question we will first need an acceptable theory of quantum gravity. (My own point of view on this topic has shifted markedly over the last few years - I was initially very hopeful that chronology protection could be proved from first principles within the context of semi-classical quantum gravity.)

Given the impossibility of proving chronology protection within the framework of semi-classical quantum gravity, and the concomitant need to address quantum gravity itself, maybe it might be a good idea to adopt chronology protection as an axiom - and let it help guide us to an acceptable theory of quantum gravity 断.

\section{ACKNOWLEDGMENTS}

This research was supported by the U.S. Department of Energy.

I wish to thank Bernard Kay and Robert Wald for their thoughtful comments.

* $\quad$ Electronic mail: visser@kiwi.wustl.edu

[1] B. S. DeWitt, "Quantum gravity: the new synthesis", in General Relativity: An Einstein Centenary Survey, edited by S. W. Hawking and W. Israel, (Cambridge University Press, Cambridge, 1979).

[2] N. D. Birrell and P. C. W. Davies, Quantum fields in curved spacetime, (Cambridge University Press, Cambridge, 1982).

[3] S. A. Fulling, Aspects of quantum field theory in curved space-time, (Cambridge University Press, Cambridge, 1989).

[4] M. Visser, Lorentzian wormholes - from Einstein to Hawking, (AIP Press, New York, 1995).

[5] M. S. Morris and K. S. Thorne, Am. J. Phys. 56, 395 (1988).
[6] M. S. Morris, K. S. Thorne, and U. Yurtsever, Phys. Rev. Lett. 61, 1446 (1988).

[7] M. Visser, Phys. Rev. D39, 3182 (1989).

[8] M. Visser, Nucl. Phys. B328, 203 (1989).

[9] S. W. Hawking, "The Chronology Protection Conjecture", in Proceedings of the 6th Marcel Grossmann Meeting, Kyoto, Japan, June 23-29, 1991, edited by H. Sato, (World Scientific, Singapore, 1992).

[10] S. W. Hawking, Phys. Rev. D46, 603 (1992).

[11] S. W. Kim and K. S. Thorne, Phys. Rev. D43, 3929 (1991).

[12] V. P. Frolov, Phys. Rev. D43, 3878 (1991).

[13] M. Visser, Phys. Rev. D47, 554 (1993)

[14] M. Visser, Nucl. Phys. B416, 895 (1994).

[15] B. S. Kay, M. Radzikowski, and R. M. Wald, Quantum Field Theory on Spacetimes with a Compactly Generated Cauchy Horizon, gr-qc/9603012; Communications in Mathematical Physics 183, 533-556 (1997).

[16] J. A. Wheeler, Phys. Rev. 97, 511 (1955).

[17] J. A. Wheeler, Ann. Phys. (NY). 2, 604 (1957).

[18] K. S. Thorne, R. H. Price, and D. A. Macdonald, (editors), Black holes: The membrane paradigm, (Yale, New Haven, 1986).

[19] S. V. Sushkov, Chronology protection and quantized fields: Complex automorphic scalar field in Misner space, gr-qc/9509056; Class. Quant. Grav. 14, 523-534 (1997).

[20] S. V. Sushkov, Quantum complex scalar field in the twodimensional space-time with closed timelike curves and a time machine problem. gr-qc/9410008; Class. Quant. Grav. 12, 1685-1698 (1995).

[21] S. V. Krasnikov, On the quantum stability of the time machine, gr-qc/9508038; Phys. Rev. D54, 7322-7327 (1996).

[22] S. V. Krasnikov, Singularity free WEC respecting time machine; gr-qc/9702031.

[23] M. Visser, Traversable wormholes: the Roman ring, grqc/9702043; Physical Review D45, 5212-5214 (1997).

[24] C. R. Cramer and B. S. Kay, Stress-energy must be singular on the Misner space horizon even for automorphic fields, gr-qc/9606027. Class. Quant. Grav. 13, L143-L149 (1996).

[25] D. G. Boulware, Quantum field theory in spaces with closed timelike curves, hep-th/9207054; Physical Review D46, 4421 (1992).

[26] J. D. Grant, Cosmic strings and chronology protection, hep-th/9209012; Physical Review D47, 2388 (1993).

[27] T. Tanaka and W. A. Hiscock, Massive scalar field in multiple connected spacetime, gr-qc/9504021: Physical Review D52, 4503 (1995).

[28] B. Kay, M. Radzikowski, and R. M. Wald, private communication.

[29] Earlier attempts at developing notions somewhat analogous to the reliability horizon can be found in 99 and 11]. These early suggestions involve an undesirable observer dependence [4].

[30] Kuo and Ford have previously suggested that fluctuations in the stress-energy tensor might invalidate the semi-classical approximation long before curvatures become large. See C.-I. Kuo and L. H. Ford, Phys Rev D47, 4510-4519 (1993). The difference here is that I am 
focussing on fluctuations in the metric, not the stressenergy. Even if stress-energy fluctuations are large this does not automatically mean that metric fluctuations must be large.

[31] R. M. Wald, General Relativity, (U. Chicago Press, Chicago, 1984), pp. 212-216, see esp. pp. 214-215. 